\title{
Diffusion-controlled first contact of the ends of a polymer: Crossover between two scaling regimes
}

\author{
Jeff Z. Y. Chen, ${ }^{1}$ Heng-Kwong Tsao, ${ }^{2}$ and Yu-Jane Sheng ${ }^{3}$ \\ ${ }^{1}$ Department of Physics, University of Waterloo, Waterloo, Ontario, Canada N2L $3 G 1$ \\ ${ }^{2}$ Department of Chemical and Materials Engineering, National Central University, Jhongli, Taiwan 320, Republic of China \\ ${ }^{3}$ Department of Chemical Engineering, National Taiwan University, Taipei, Taiwan, 106, Republic of China
}

(Received 10 May 2005; published 8 September 2005)

\begin{abstract}
We report on Monte Carlo simulations of loop formation of an ideal flexible polymer consisting of $N$ bonds with two reactive ends. We determine the first-passage time associated with chain looping that yields a conformation in which the end monomers are separated by a distance $a$ - the reaction radius. In particular, our numerical results demonstrate how this time scale crosses over from $\tau_{\text {first }} \sim N^{3 / 2} / a$ to the $a$-independent $\tau_{\text {first }}$ $\sim N^{2}$ as $N$ is increased. The existence and characteristics, of the two scaling regimes and the crossover between the two, are further illuminated by a scaling argument.
\end{abstract}

DOI: 10.1103/PhysRevE.72.031804

PACS number(s): 82.35. $-\mathrm{x}, 61.41 .+\mathrm{e}$, 05.70.Ln

\section{INTRODUCTION}

Loop formation of a polymer chain is a dynamic process by which two monomers along the chain approach each other within a small distance. Subsequently, the interaction of these two monomers occur as the result of the normally short-ranged interaction (with a force range $a$ ) between the reactive monomers. The locations of these interacting monomers, along the contour of the polymer chain, could be as distant as the entire chain length in the case of two interactive ends. Starting from an open configuration where the two reactive monomers are separated by a physical distance that could be much greater than $a$, the polymer undergoes configurational fluctuations that bring together (or separate) the two ends-which is a process that is solely determined by the entire chain.

A biopolymer, for example, may require loop formation as a primary step for acquiring a desired structure to perform its biological functioning. Understanding the looping dynamics of a relatively simple system that depends on a few essential physical parameters can form a first step toward gaining much insight into a wide range of biological and physical processes such as protein folding [1] and DNA replication [2]. The recent advance in single-molecule manipulations on this kind of systems has allowed one to probe chain closing times [3]. In reality, a biopolymer can carry many reactive groups and the reaction between two groups are usually further complicated by the participation of other molecules in the system. There are also examples in synthetic polymers where loop formation of a polymer is an important process [4].

On the theoretical side, much effort has been paid to studying single-loop formation of a polymer with two reactive ends [5-14]. One of the main issues is whether or not the diffusion-controlled reaction of two ends is more complicated than the dynamic behavior represented by the autocorrelation function of the end-to-end vector. Of particular interest is the characteristic time that required for the two ends to approach each other and react. In the case of instantaneous reaction, the focal point has been on the mean first-passage closing time, $\tau_{\text {first }}$, for the reactive monomers to close within a distance of $a$ from each other for the first time starting from an open configuration (averaged over all initial conformations that are typically assumed to follow an equilibrium distribution). The closing dynamics in this case could be complicated by the internal dynamic modes, which lead to the rapid motion of chain ends [5]. The competition, between local equilibration at the length scale $a[7,11]$ and the global conformation fluctuations, gives rise to more than one scaling regimes for $\tau_{\text {first }}$. To complicate things further, a typical theoretical approach to this problem usually relies on making several approximations $[5-7,11,12]$. For example, the assumption of "local equilibrium" by Szabo, Schulten, and Schulten (SSS) leads to

$$
\tau_{\text {first }}=\tau_{\text {SSS }} \sim N^{3 / 2} / a
$$

for an ideal flexible chain when $a$ is small [7]. On the other hand, by keeping $a / b$ finite, it has been argued that for a long ideal flexible polymer, $\tau_{\text {first }}$ has another scaling behavior $[5,6]$

$$
\tau_{\text {first }}=\tau_{\mathrm{R}} \sim N^{2},
$$

where $\tau_{\mathrm{R}}$ is the Rouse relaxation time representing the global relaxation of the polymer, which scales as $N^{2}$ [15]; note that $\tau_{\mathrm{R}}$ does not depend on $a$.

The seeming discrepancy between $\tau_{\mathrm{SSS}}$ and $\tau_{\mathrm{R}}$ has also inspired numerical studies in an effort to understand the difference between the two. However, earlier simulations were limited to a relatively small parameter space and the results are not conclusive. The computer simulations of Paster, Zwanzig, and Szabo confirmed the $N^{3 / 2}$ dependence of $\tau_{\text {SSS }}$ for a single value of $a$, leaving the inverse $a$-dependence unchecked [11]; furthermore, if they had extended their simulations for the exactly same $a$ to a much larger $N$, they would have seen the crossover to a different scaling behavior. In contrast, the simulations of Podtelezhnikov and Vologodskii exhibited the $N^{2}$ dependence of $\tau_{\mathrm{R}}$, but the results also suggested an $a$-dependent coefficient that does not exist in $\tau_{\mathrm{R}}[16]$. In a theoretical treatment supplemented by simu- 
lations, Portman showed that $\tau_{\mathrm{SSS}}$ and the mean first-passage time, determined according to the Wilemski and Fixman approximation, are the lower and upper bonds for $\tau_{\text {first }}$, respectively [17]. Because $\tau_{\mathrm{R}}$ is one of the predictions based on the Wilemski and Fixman approximation, this indicates the possible existence of the crossover between the two types of scaling behavior in Eqs. (1) and (2) [11].

The main purpose of this paper is threefold, first we provide extensive computer simulations of polymer loop formation in a wide parameter range. The results can be used to provide numerical evidences convincingly supporting the two scaling regimes (see Sec. III A) and demonstrating the crossover between these (see Sec. III B). This is computationally demanding and the current computational power has just allowed us to consider sufficiently large $N$ and relatively small $a$. For consistency with the physical models used in most previous theoretical treatments, we model a polymer as a free-jointed chain with no excluded volume interaction or hydrodynamic effects. In particular, we study how distinct scaling regimes of $\tau_{\text {first }}$ emerge. Our simulations show a crossover from $\tau_{\mathrm{SSS}}$ to the $a$-independent $\tau_{\mathrm{R}}$ at certain values of $N$ and $a$. Unlike earlier simulations, our simulations provide direct evidence of the crossover.

Second, we also give a scaling argument for the observed crossover between the two scaling regimes (see Sec. III C). The theory is conceptually based on a simple physical picture, without the involvement of approximations and complex mathematical derivations used previously to arrive at the two scaling relations, in Eqs. (1) and (2). We derive the relevant time scales and length scales that are responsible for the competitions between the two scaling regimes, in consistence with existing theories of polymer dynamics.

Finally, we examine the conditional distribution functions of $\tau_{\text {first }}$ with a fixed initial end separation $R$ for two typical values of $a$. The analysis further clarifies the physical picture of crossover between the SSS anf Rouse regimes (see Sec. III D).

\section{MODEL AND SIMULATION DETAILS}

The polymer model used in this study is a typical freely jointed chain, consisting of $N$ bonds of fixed length $b$ [15]. In a simulation implementation, a randomly selected monomer is rotated about the axis defined by the vector connecting the two nearest-neighbor monomers; the lengths of the connected bonds are unaffected by the rotation. The rotational angle was selected as a random number between $(-\delta,+\delta)$, where $\delta=\delta_{0} \equiv \pi / 20$ [18]. All time scales in this work are measured in terms of a MC step (MCS); within each MCS all monomers along the chain have the probability to move once.

An initial configuration is generated by a random walk with step length $b(=1)$. The chain is then subject to $\mathrm{MC}$ moves. The terminal monomers, labeled 0 and $N$, are considered to have made a contact when they fall within the reactive range, $R \leqslant a$, where $R$ is the end-to-end distance. In the diffusion-controlled reaction, the two ends are assumed to instantaneously react and trap each other (i.e., the chain closes) as soon as $R \leqslant a$; the simulation for a single closing event then stops. A first-passage time, $t_{\text {first }}$, the time for the chain to close for the first time from a given initial conformation, is then recorded. We repeat this simulation with a new initial conformation generated by a fresh random seed every time. For each set of $[a, N]$, a total of $M=400$ closing events have been observed, with the exception of the data points for $N>300$ where $M=50$. The average first-passage time $\tau_{\text {first }}$ is then an algebraic average of $t_{\text {first }}$ observed in these $M$ events.

\section{RESULTS AND DISCUSSION}

\section{A. Mean first-passage time for chain closing}

Figure 1(a) shows the simulation data of $\tau_{\text {first }}$ as a function of $N$ for various values of $a$. To examine the $N$ dependence of $\tau_{\text {first }}$, we have replotted the same data set in Fig. 1(b), where the scaled first-passage time $\tau_{\text {first }} / N^{2}$ is displayed as a function of $\sqrt{N} b / a$ (i.e., the ratio of the root-mean-square end-to-end distance, $R_{0}$, to the interaction radius) for various choices of $a / b$. As shown in the figure, data points corresponding to different values of $a / b$ converge to a constant for large $N$, implying that $\tau_{\text {first }} / N^{2}$ is not dependent on $N$, nor on $a / b$, in the limit of $\sqrt{N} b \gg a$. Hence,

$$
\tau_{\text {first }} \propto N^{2} \quad(\sqrt{N} b \gg a) .
$$

This numerically verifies that the scaling behavior of $\tau_{\text {first }}$ is the same as that of the Rouse time in this limit.

While this seems to be straightforward, it has not been fully confirmed numerically in the past for some reasons. For example, earlier Brownian dynamics simulations of $\tau_{\text {first }}$ were limited to $N \leqslant 55$ due to computational limitations and failed to demonstrate the $a$-independent asymptotic result in Eq. (3) [16] [see also the apparent $a$-dependence of some intermediate values of $N$ in Fig. 1(b)]. The $a$-independence of Eq. (3) implies that the first-passage time of closing, of a sufficiently long chain, is independent of their force range $a$. This can be contrasted with the action time between lowmolecular particles in the diffusion limited case where the action time varies inversely with their reactive range. Unlike low molecular systems, long-chain molecules show unique dynamics due to the presence of conformation fluctuations represented by, for example, i.e., the Rouse modes [15]. The Rouse time is a characteristic time that describes the fluctuations of the chain ends; on average, it takes $\tau_{\mathrm{R}}$ for the chain ends to have a chance to reach the vicinity of each other in passing. The question then becomes would the two ends have a chance to reach a distance of $a$ (which could be small) during $\tau_{\mathrm{R}}$ ? As will be demonstrated in Sec. III C, if the answer is yes, $\tau_{\mathrm{R}}$ is the characteristic time for the entire process.

On the other hand, another distinctively different scaling regime can be exhibited by our data for moderate $N$ with very small $a / b$. In order to perform a careful analysis, we recall that the assumption of local equilibrium led SSS to obtain [7] 

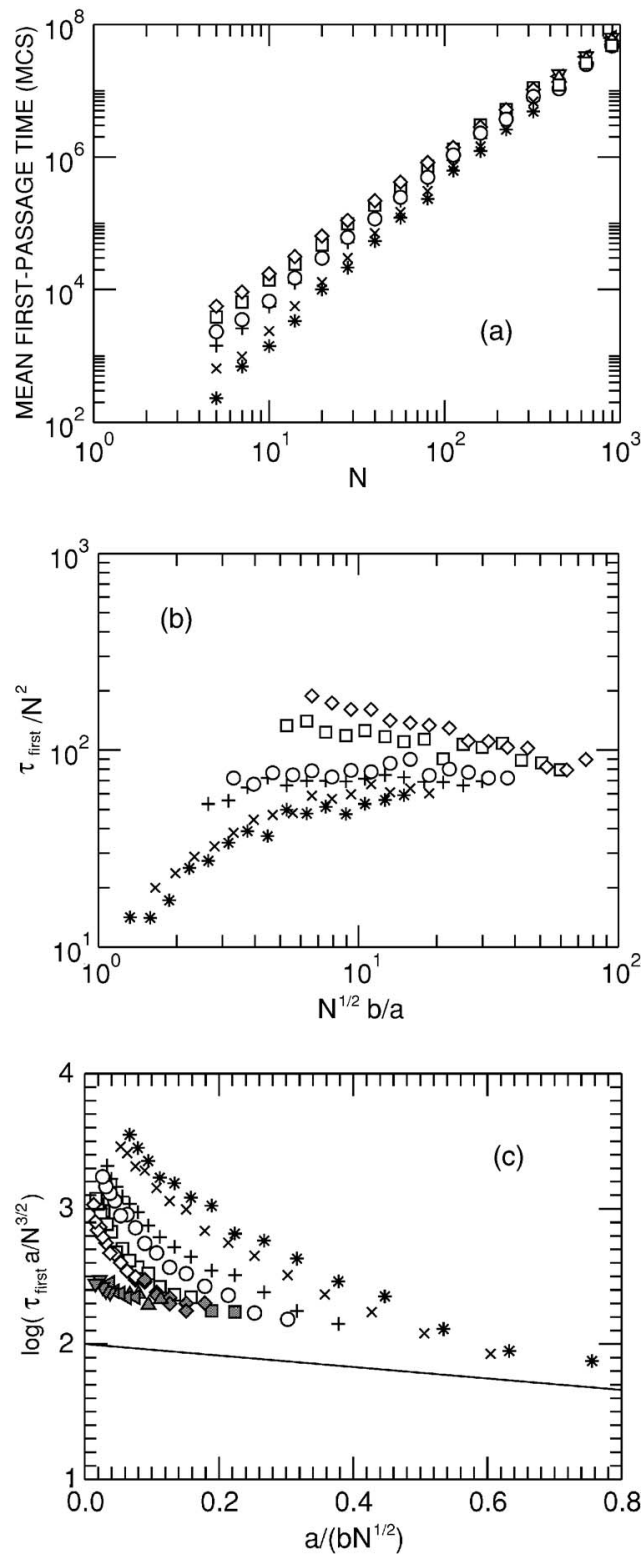

FIG. 1. Mean first-passage time $\tau_{\text {first }}$ for closing. Stars, crosses, plus signs, circles, squares, diamonds represent MC simulation data for $a / b=2.0,1.6,1.0,0.8,0.5$, and 0.4 , respectively. In plot (a), the original measurements are displayed; in (b), scaled $\tau_{\text {first }} / N^{2}$ is shown as a function of the ratio $R_{0} / a$ where $R_{0}$ is the root-meansquare end-to-end distance, i.e., $R_{0}=b \sqrt{N}$; (c) is a plot of $\tau_{\text {first }} a / N^{3 / 2}$ vs $a / R_{0}$. In (c), our data have been supplemented with an additional MC data set for smaller values of $a / b(=0.4,0.3,0.2$, and 0.1$)$ (diamonds, up triangles, left triangles, and down triangles, respectively). The typical errors of the data are smaller than the sizes of symbols in plot (a).

$$
\tau_{\text {first }} \propto \frac{N^{3 / 2}}{a}\left[1+\left(\sqrt{\frac{6}{\pi}}(\ln 2-1)\right) \frac{a}{b N^{1 / 2}}+\cdots\right] .
$$

In Fig. 1(b), the leading term would project a

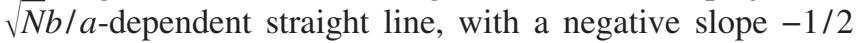
on the double-logarithmic plot. The diamonds and squares $(a / b=0.4$ and 0.5$)$ in the vicinity of $\sqrt{N} b / a \cong 10$ indeed dis- play a similar behavior, but cannot be used to make a conclusive illustration. A better perspective can be gained if we plot these data points in a different way; to illustrate a scaling relation that would follow Eq. (4), we consider $\tau_{\text {first }} a /\left(b N^{3 / 2}\right)$ as a function of $a /(b \sqrt{N})$ in a semilogarithmic plot [see Fig. 1(c)]. Equation (4) would then predict a straight line with a slope given by $\sqrt{6 / \pi}(\ln 2-1)$ for small values of $a /(b \sqrt{N})$. Because the SSS regime is only valid for very small $a / b$ (see next section), the same data sets from Figs. 1(a) and 1(b), now plotted in Fig. 1(c), are not adequate for clear demonstration of the SSS scaling. We have conducted additional MC simulations of systems with smaller values of $a / b(=0.4,0.3,0.2,0.1)$; the resulting $\tau_{\text {first }}$ have been plotted in Fig. 1(c) as filled symbols. These filled symbols can be seen to approach a constant as $a /\left(b N^{1 / 2}\right)$ decreases, which confirms the existence of the SSS limit. Moreover, the solid line in Fig. 1(c) gives an exact slope $\sqrt{6 / \pi}(\ln 2-1)$, representing the coefficient of the firstcorrection term in Eq. (3). Hence, both leading terms in Eq.

(4) can be verified by our numerical data.

Note that in order to properly simulate the Brownian motion of the polymer in solution by using a Monte Carlo approach, the simulated displacement of a monomer must be much smaller than the length scale that we are observing. The reduction of the displacement would also prompt much longer computational time. To ensure that the simulated displacement of a monomer is smaller than $a / b$, we have used $\delta=\delta_{1} \equiv 0.01$ as the maximal value for the random angle selection in an MC move for $a / b=0.3,0.2$, and 0.1 . This selection can be compared to $\delta=\delta_{0} \equiv \pi / 20$ used above for $a / b \geqslant 0.4$. A major consequence of using a different value of $\delta$ is that the units of time are now different: the simulation time it takes, in terms of MCS, to observe the same system for a defined period of time, is now approximately $\left(\delta_{0} / \delta_{1}\right)^{2}$ longer when $\delta=\delta_{1}$ is used. Hence, the first-passage times from these supplemented simulations have been multiplied by a factor of $\left(\delta_{1} / \delta_{0}\right)^{2}$ in Fig. 1(c), in order to take into account the adjustment of units.

\section{B. Crossover between the Rouse and SSS regimes}

Having confirmed that both Rouse and SSS regimes indeed exist and can be associated with the respective scaling behavior, we now turn to classifying the corresponding regimes in the parameter space. Because two different time scales are competing with each other for a given system, $\tau_{\mathrm{R}} \sim N^{2}$ and $\tau_{\mathrm{SSS}} \sim N^{3 / 2} b / a$, the first-passage time for closing is dictated by the longer of the two [11].

In the regime of the parameter space where $\tau_{\mathrm{R}} \leqslant \tau_{\mathrm{SSS}}$, or $N^{2} \leqslant c N^{3 / 2} b / a$, the first passage time follows the SSS behavior, where we have introduced a dimensionless numerical constant $c$ in the last equation for more precise specification. This inequality hence yields the crossover condition,

$$
a N^{1 / 2} / b \leqslant c .
$$

This condition, along with the requirement that we are dealing with a long-chain polymer, 


$$
a /\left(b N^{1 / 2}\right) \ll 1
$$

specifies the SSS regime. The latter essentially comes from the fact that the correction terms in Eq. (4) should be small in comparison with the leading scaling term. These two conditions are quite restrictive; on the one hand, by combining these two inequalities, we obtain

$$
(a / b)^{2} \ll c,
$$

which is an indication that only small $a / b$ systems would be expected to display the SSS scaling, consistent with the argument made by SSS themselves [7]. On the other hand, the combination of these inequalities also leads to

$$
a / b \ll \sqrt{N} \leqslant c b / a,
$$

where the two bounds have been set for $N$ : the bound of $a / b \ll \sqrt{N}$ that can be easily satisfied in most polymer systems and the other bound of $\sqrt{N} \leqslant c b / a$ that restricts the SSS scaling to the intermediate values of $N$-not all small $a / b$ systems would display the SSS scaling. In fact, for every long polymer where $\sqrt{N} \geqslant c b / a$, we recover the Rouse regime even for small $a / b$.

The filled symbols in Fig. 1(c) represent data points corresponding to $a N^{1 / 2} / b \leqslant 1.5$ where $c=1.5$ is an empirically selected constant, used for the purpose of illustrating the SSS regime. As $N$ is increased [hence lowering $a /(b \sqrt{N})$ ], the systems begin to move away from the SSS regime and the data points start to deviate from the scaling behavior.

How important is the SSS regime in real systems, in view of the restrictive conditions for validity in Eq. (8)? For most flexible chains, the force range of interaction $a$ is of the same order of magnitude as the polymer bond length $b$. As the result, $\sqrt{N}$ can easily exceed $b / a$. The Rouse time is probably the only characteristic time observable in real flexible polymers, according to the criterion for the validity of the SSS regime in Eq. (8). However, in wormlike polymer systems, the persistent length (equivalent to $\frac{1}{2} b$ ) could be much larger than a typical interaction range $a$. Double-stranded DNA molecules are good examples of such systems. The SSS regime then becomes significant [13]. We have recently directly observed the SSS scaling for the first passage time of closing in wormlike chains by numerical simulations [14].

\section{Scaling argument for the crossover between the Rouse and SSS regimes}

So far, we have provided the numerical evidences for the existence of and the crossover between the Rouse and SSS regimes. The previous theoretical approaches used to arrive at these results, however, are highly mathematical [5-7,11], and the approximations used are not always transparent [12]. In this section, we develop a simple and consistent physical picture through a scaling argument [19], that supports both $\tau_{\mathrm{R}}$ and $\tau_{\mathrm{SSS}}$.

Consider the end-to-end vector of the polymer chain. The global dynamic behavior of this vector is governed by the first normal mode of the entire polymer [15], which projects the slowest relaxation time (the Rouse time); the tip of the vector, however, undergoes rapid Brownian motion within a small region much smaller than $R_{0}$, the root-mean-square end-to-end vector. In particular, we write $\Delta$ for the amount of time that it takes to explore a regional space of dimension $a$ (assumed here $\leqslant a_{R}$ to be defined below) by the tip of endto-end vector. Now, because the dynamic motion is diffusive,

$$
\Delta \approx a^{2} / D_{0}
$$

where $D_{0}$ is the effective diffusion constant associated with the end monomer. Within such a small region and during $\Delta$, the Brownian motion of the end-to-end vector tip contains the fastest normal modes of the polymer chain; another way of viewing this is that, during this period of time, the end monomer "feels" the presence of a small number of connected neighboring monomers only, not the entire chain. Thus, $D_{0}$ is proportional to $k_{B} T / \zeta$ (the Einstein relation) where $\zeta$ is the friction constant experienced by the end monomer.

Now, during the Rouse time, a much larger time scale than $\Delta$, the end-to-end vector fluctuates globally, covering the entire space of dimension $R_{0}$. In total, the volume $R_{0}^{3}$ contains $K$ small regions, each having volume $a^{3}$, where $K$ $=R_{0}^{3} / a^{3}$. In order to find each other starting from a typical distance of $R_{0}$, the two polymer ends must explore the entire $R_{0}^{3}$ volume thoroughly (i.e., searching those small regions of volume $a^{3}$ ). Hence, the time that it takes to do this is

$$
\tau \propto K \Delta \propto \frac{R_{0}^{3}}{a^{3}} \frac{a^{2}}{D_{0}} \propto \frac{b}{a} N^{3 / 2}\left[\frac{b^{2}}{D_{0}}\right],
$$

which gives a simple explanation of the SSS time in Eq. (1), within the definition of the units of time represented by the quantity in the square brackets of the above equation. This expression is identical to Eq. (29) in Ref. [11].

This scaling argument is based on the assumption that Eq. (10) is greater than the Rouse time, $\tau_{\mathrm{R}} \sim N^{2}$ - the polymer ends need to find each other in the vicinity (governed by the Rouse time), before they can thoroughly explore the region of a grain size $a^{3}$. Therefore, $\tau_{\mathrm{R}}$ is the lower bound for $\tau_{\text {first }}$, the first-passage time of reaction between the two ends. Returning to the discussion in the second paragraph of Sec. III B, we see that the SSS regime is restricted to the parameter space given by Eq. (8).

Another interesting question is, during the Rouse time, how much detail in space would the two polymer ends explore. The answer to this question is actually already contained in Eq. (10). Equating the left-hand side of the equation with the Rouse time $\tau_{\mathrm{R}}$, we can take $a$ on the right-hand side as the dimension of the space, $a_{R}$, explored by the polymer ends during the Rouse time. This yields

$$
a_{R} \propto b / \sqrt{N}
$$

which is a dimension much smaller than the Kuhn length $b$. In light of the above discussion, the crossover condition [Eq. (5)] can be related to the physical meaning of $a_{R}$ as well; when 


$$
a \leqslant a_{R} \quad(\mathrm{SSS}),
$$

the chain ends could not find each other within the distance $a$, and must continue the search beyond the time period $\tau_{\mathrm{R}}$; when

$$
a \geqslant a_{R} \quad \text { (Rouse) }
$$

the chain ends have already found each other during the Rouse time $\tau_{\mathrm{R}}$. Equation (12) is consistent with the righthand side of Eq. (8).

As a final note, $a_{R}$ is also a characteristic length scale at which we need to consider all Rouse modes, fast and slow, for the description of the motion of end-to-end vector tip [5]. This can be contrasted with two limiting cases. First, when we consider the dynamical properties at a length scale $a$ that is much smaller than $a_{R}$ during time $\Delta$ (in the SSS regime), only the fastest Rouse modes contribute to the dynamics. Second, when we consider the "global" dynamic properties of the polymer at a length scale $R_{0}$ that is much greater than $a_{R}$, only the slowest Rouse mode contribute to the dynamics [15]. Hence, $a_{R}$ is a characteristic length scale that reflects the division between the fast and slow Rouse modes in polymer dynamics.

\section{Distribution function of $t_{\text {first }}$}

The physical picture presented above can be further augmented by the distribution function of the first passage time of closing. To provide sufficient statistical data for a distribution-function analysis, we have concentrated on two cases (a) $a / b=0.4$ and (b) $a / b=1.6$ for $N$ varying from $N$ $=10$ to $N=80$. As can be viewed from the two scaling plots of Fig. 1, systems (a) and (b) belong to the near-SSS and Rouse regimes, respectively, for the mentioned $N$ values. In total, $10^{6}$ independent closing events, represented by $t_{\text {first }}$, have been recorded for the analysis performed in this section. The histogram of $t_{\text {first }}$ is then plotted in Fig. 2.

Wilemski and Fixman [6] suggested that the distribution function for $t_{\text {first }}$ should become simple exponential, $f\left(t_{\text {first }}\right)$ $\propto \exp \left(-t_{\text {first }} / \tau_{\text {first }}\right)$, in the large $t_{\text {first }}$ limit. Indeed, all open symbols for the near-SSS regime $(a / b=0.4)$ in Fig. 2 form a straight line in a semilogarithmic plot, consistent with the suggested exponential form. The distribution functions for the four values of $N$, however, are different in the Rouse regime, $a / b=1.6$, where the short-time behavior, in a relatively significant region in $t_{\text {first }} / \tau_{\text {first }}$, displays the nonexponential form; the distribution function contains a fast initial decay, indicating more short- $t_{\text {first }}$ events.

Where do these short- $t_{\text {first }}$ events come from? To further dissect the physical picture, we have examined the mean first-passage time for closing $\left\langle t_{\text {first }}\right\rangle(R)$ as a function of an initial end-to-end distance $R$. Computationally, to obtain the distribution function discussed above, $10^{6} \mathrm{MC}$ dynamic trajectories have been produced, with the initial configurations randomly adopted from independent equilibrium states. As such, the initial end-to-end distance follows an equilibrium density distribution function that are very close to Gaussian - the unnoticeable deviation comes from the discrete nature of the adopted simulation model. We selected all

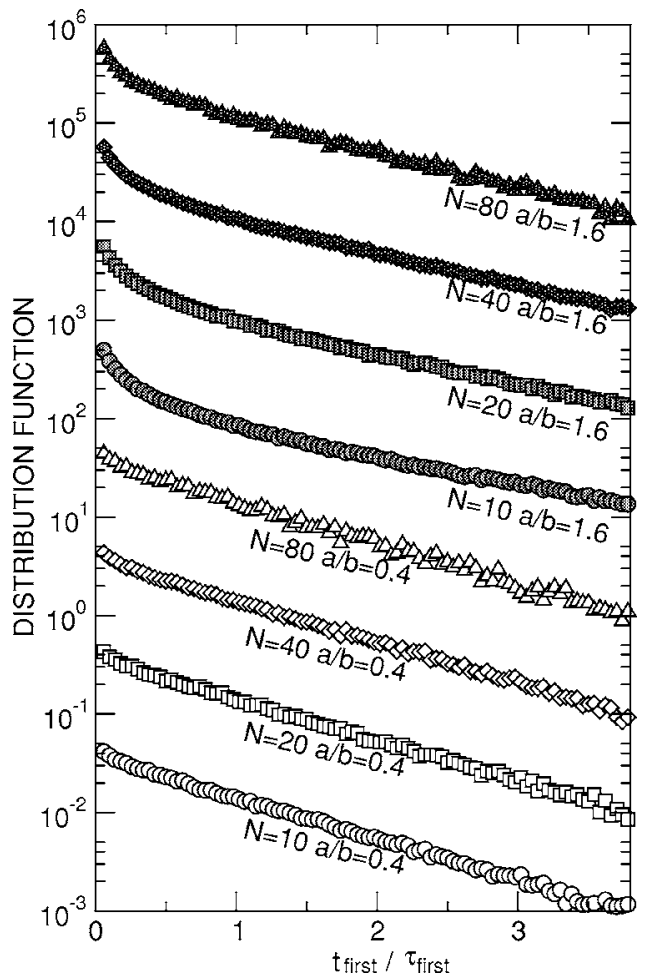

FIG. 2. The distribution function of the first-passage time $t_{\text {first }}$ in a near-SSS (open symbols) and Rouse (shaded symbols) regimes for various $N$. To clearly display the curves, each set of data in this plot has been shifted by a factor of 10 from the lower neighboring set. The errors of the data are similar to the sizes of the plotted symbols.

trajectories with a matching initial end-to-end distance to a prespecified $R$ range for this part of the analysis. $\left\langle t_{\text {first }}\right\rangle(R)$ as a function of $R$ for both (a) $a / b=0.4$ and (b) $a / b=1.6$ is displayed in Fig. 3 in a reduced form. In the Rouse regime, we are dealing with systems where the capturing radius, $a$, is relatively large in comparison with $R_{0}$. Events that start with an $R$ shorter than $R_{0}$ contain two possible types of trajectories, those with the ends to separate farther from each other and those with the ends to approach closer to each other. Because of the relatively large $a$, the probability, that the two ends capture each other on a simple path consisting of mainly the approaching motion, is much greater than that in smaller $a$ systems. These short- $t_{\text {first }}$ events are particularly more frequent in systems with smaller $N$ (or, larger $a / R_{0}$ ). Hence, on average, for a fixed $R \leqq R_{0}$ to start with, smaller $N$ systems produce a smaller $\left\langle t_{\text {first }}\right\rangle(R)$, which can be seen in Fig. 3(b). These short- $t_{\text {first }}$ events significantly contribute to the initial, fast-decaying portion of the distribution functions of $a / b=1.6$ systems in Fig. 2.

Now, in small $a / b$ systems (in the SSS regime), these short- $t_{\text {first }}$ events are much rarer. This is reflected by another dominating feature in Fig. 3: the reduction of the overall variation of $\left\langle t_{\text {first }}\right\rangle(R) / \tau_{\text {first }}$ as $a / b$ decreases from 1.6 to 0.4 (note the two different vertical scales). The spreading of the data points in the vicinity of $R^{2} / N \approx 1$ in Fig. 3(b) for $a / b$ $=1.6$ can be contrasted with the narrow range of variations in Fig. 3(a) for $a / b=0.4$. As can be projected based on the 

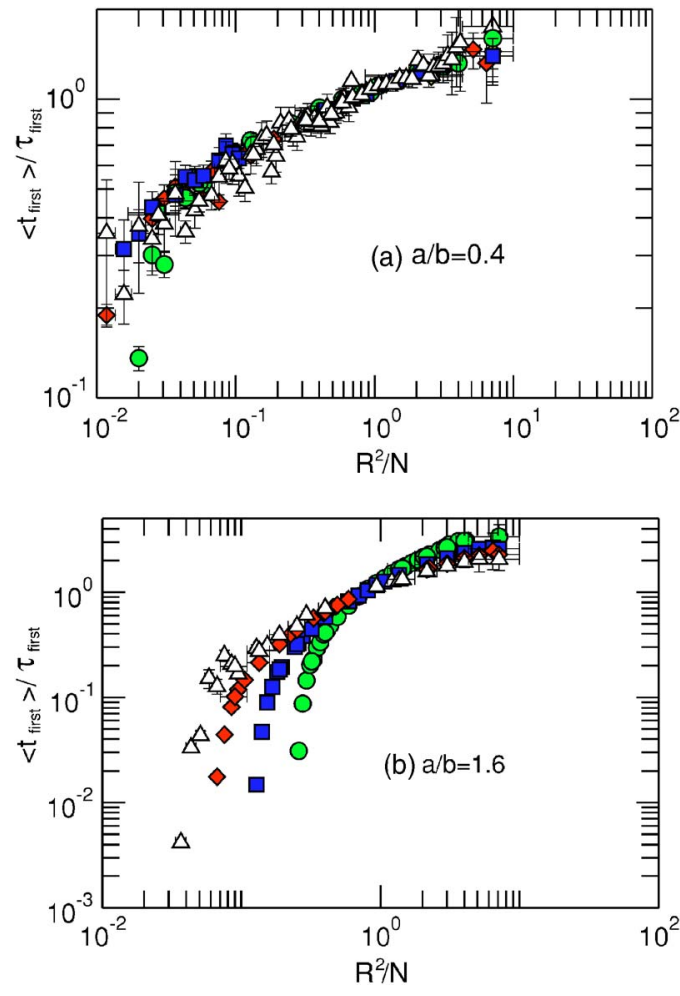

FIG. 3. (Color online) Average first-passage closing time as a function of the initial square end-to-end distance $R^{2}$ for (a) $a / b$ $=0.4$ (close to the SSS regime) and (b) $a / b=1.6$ (in the Rouse regime). Circles, squares, diamonds, and triangles correspond to $N=10,20,40$, and 80 .

general trend, a further decreasing of $a / b$-bringing the system to the "true" SSS region-would yield a similar plot to Fig. 3(a) where $\left\langle t_{\text {first }}\right\rangle_{R} / \tau_{\text {first }}$ becomes a constant $(\approx 1)$. This asymptotic limit can be understood from the same explanation in the above paragraph. For small $a / b$, regardless of the initial distance between the ends to start with, the two ends are very likely to miss each other in an end-approaching event. Instead, the chain would relax from an initial end-toend separation of $R$ to $R_{0}=\sqrt{N} b$, as the first step. This process takes up $\tau_{\mathrm{R}}$ on average. The first passage time, of closing for two ends to start with a separation of distance $R_{0}$, is $\tau_{\mathrm{SSS}}$ in the SSS regime; this fact, together with $\tau_{\mathrm{SSS}} \gg \tau_{\mathrm{R}}$ in the SSS regime, would completely undermine the initial relaxation period from $R$ to $R_{0}$. Hence, $\left\langle t_{\text {first }}\right\rangle(R) / \tau_{\text {first }}$ should become an $R$ - and $N$-independent constant in the SSS regime. This independence can be explained by the reduced physical picture of diffusion between two end monomers [13] in an effective spring potential, after the internal degrees of freedom being integrated out. In the so-called "overdamped" limit, the effective free energy barrier, that two monomers must overcome before approaching to each other in a distance smaller than $a$, is much greater than $k_{B} T$; the time duration for the reaction to happen is largely dependent on the overall barrier height and is not sensitive to the initial separation of the two monomers [20].

To further contrast looping formations operating in the SSS and Rouse regimes, we have also considered the (normalized and conditional) distribution of $t_{\text {first }} / \tau_{\text {first }}$ for a given
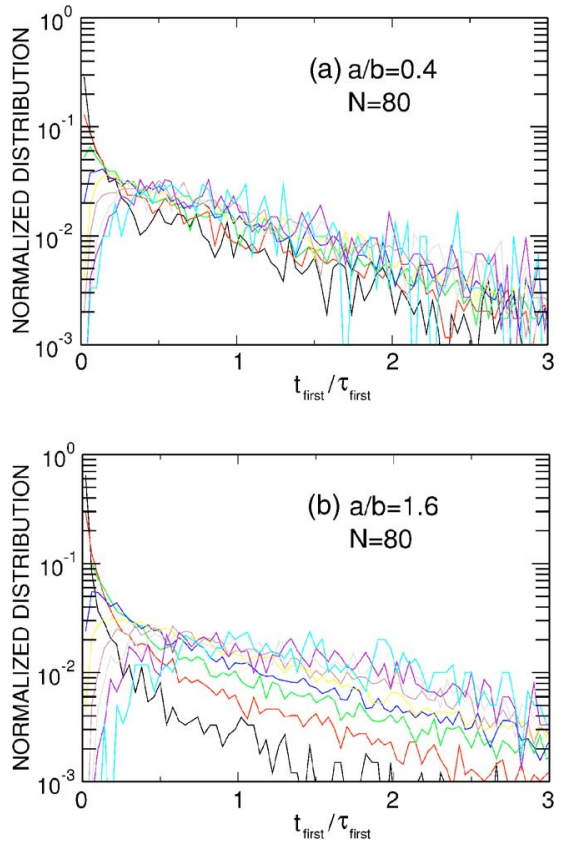

FIG. 4. (Color online) The normalized conditional distribution functions of the first-passage time for fixed initial end-to-end separation for $N=80$ in the (a) SSS and (b) Rouse regimes. In each plot, from top to bottom near $t_{\text {first }}=0$, curves correspond to $R / \sqrt{N}$ $=[0.2,0.4],[0.4,0.6],[0.6,0.8],[0.8,1.0],[1.0,1.2], \quad[1.2,1.4]$, $[1.4,1.6]$, and $[1.6,1.8]$, respectively. The fluctuations of the curves are good reflections of the error sizes of the data.

$R$. In the Rouse regime [see Fig. 4(b)], the distribution functions have a strong $R$ dependence, as we discussed above, reflected in these plots by the different slopes of the distribution function in the semilogarithmic plots. The events that start with a smaller $R$ have significant statistics which are different in the initial period $t_{\text {first }} / \tau_{\text {first }} \ll 1$. Because the chain ends are started with already close distance, the reaching of $R=a$ could happen in the "first" passage near the vicinity of $R=a$ within a relatively short time scale. A very interesting limit is the large $t_{\text {first }}$ behavior of the distribution functions for large $R$ 's, which tend to approach a common slope. Note these starting distances are relatively far from $R_{0}$, the relaxation from these positions to $R_{0}$ takes almost the same $\tau_{\mathrm{R}}$. Sokolov has recently analyzed the distribution function in Fig. 4 by using a much more complicated mathematical procedure [12].

In the near-SSS regime [see Fig. 4(a)], all distribution functions, no matter the values of $R$, collapse into a universal, simple exponential decay at large $t_{\text {first }}$. This represents the events that are characterized by the looping of the polymer chain from $R_{0}$ to a distance $a$. Because $a$ is small, most trajectories must spend multiple number of passes in the vicinity of $R=a$ to thoroughly explore the regime. The number of passes, estimated in Secs. III B and III C, could be as large as $\tau_{\mathrm{SSS}} / \tau_{\mathrm{R}} \approx b /(a \sqrt{N})$, which is actually a large, $R$-independent number in the SSS regime [see Eq. (6)].

\section{SUMMARY}

In summary, we have studied the closing dynamics of a polymer with two reactive ends, using both Monte Carlo 
simulations and a scaling argument. The main focus has been put on clarification of the crossover between the two scaling regimes. We have performed carefully prepared Monte Carlo simulations that sampled a fairly large parameter space and accumulated significant statistics for this purpose. This has allowed us to convincingly pin down the scaling regimes and the crossover between them. We have shown how the first passage time $\tau_{\text {first }}$ of closing crosses over from the $a$-dependent $\tau_{\mathrm{SSS}} \sim N^{3 / 2} / a$ to the $a$-independent $\tau_{\text {Rouse }} \sim N^{2}$ as $N$ and $a$ varies. Within a scaling argument, we have derived the observed scaling relationship between $\tau_{\mathrm{SSS}}$ and $N$ as well as $a$, directly from a simple physical picture. The same reasoning has also allowed us to determine the various quantities that can be further used to characterize the polymer dynamics.

\section{ACKNOWLEDGMENTS}

The authors wish to acknowledge the financial support from NSERC, the computational time allocation from Sharcnet, and the critical reading of an earlier version of this paper by Bae-Yeun Ha.
[1] H.-X. Zhou, J. Chem. Phys. 118, 2010 (2003).

[2] S. Jun, Ph.D. thesis, Simon Fraser University, 2004.

[3] N. L. Goddard, G. Bonnet, O. Krichevsky, and A. Libchaber Phys. Rev. Lett. 85, 2400 (2000).

[4] See, for example, M. A. Winnik, in Cyclic Polymers, edited by J. A. Semlyen (Elsevier, New York, 1986).

[5] M. Doi, Chem. Phys. 9, 455 (1975).

[6] G. Wilemski and M. Fixman, J. Chem. Phys. 60, 866 (1974); 60, 878 (1974).

[7] A. Szabo, K. Schulten, and Z. Schulten, J. Chem. Phys. 72, 4350 (1980).

[8] B. Friedman and B. O'Shaughnessy, Phys. Rev. Lett. 60, 64 (1988); Phys. Rev. A 40, 5950 (1989).

[9] A. Perico and M. Beggiato, Macromolecules 23, 797 (1990).

[10] B. Friedman and B. O'Shaughnessy, Macromolecules 26, 4888 (1993).

[11] R. W. Pastor, R. Zwanzig, and A. Szabo, J. Chem. Phys. 105, 3878 (1996).

[12] I. M. Sokolov, Phys. Rev. Lett. 90, 080601 (2003).
[13] S. Jun, J. Bechhoefer, and B.-Y. Ha, Europhys. Lett. 64, 420 (2003).

[14] J. Z. Y. Chen, H.-K. Tsao, and Y. J. Sheng, Europhys. Lett. 65, 407 (2004).

[15] M. Doi and S. F. Edwards, The Theory of Polymer Dynamics (Oxford University Press, New York, 1988).

[16] A. Podtelezhnikov and A. Vologodskii, Macromolecules 30, 6668 (1997).

[17] J. J. Portman, J. Chem. Phys. 118, 2381 (2003).

[18] The magnitude of $\delta$ affects the computer simulation rate. A large $\delta$ yields a large displacement of monomers, thus less total MCS for the polymer model to fluctuate from one configuration to another. However, the selection of $\delta$ is constrained by the fact that the corresponding displacement of monomers of each move should be much less than the force range $a$.

[19] P.-G. De Gennes, Scaling Concepts in Polymer Physics (Cornell University Press, Ithaca, NY, 1993).

[20] P. Hänggi, P. Talkner, and M. Borkovec, Rev. Mod. Phys. 62, 251 (1990). 